\title{
Condiciones de trabajo, fatiga laboral y bajo peso al nacer en vendedoras ambulantes*
}

\author{
Patricia Hernández-Peña, M.C., M.S.P., ${ }^{(1)}$ María de la Luz Kageyama, M.C ., M.P.H. .(2) Irma Coria, M.C. .(1)
} Bernardo Hernández, Psic., Dr. Sc., ${ }^{(4)}$ Sioban Harlow M.D., Dr. Sc. ${ }^{(5)}$

\section{Hernández-Peña P, Kageyama ML, Coria I, Hernández B, Harlow S. Condiciones de trabajo, fatiga laboral y bajo peso al nacer en vendedoras ambulantes. Salud Publica Mex 1999;41:101-109.}

\section{Resumen}

Objetivo. Este trabajo tipifica las condiciones demográficas, socio económicas, laborales, de fatiga laboral y daño reproductivo en una población femenina en edad fértil que trabaja en la venta ambulante en las calles de la Ciudad de México. Material y métodos. Se entrevistaron 426 vendedoras ambulantes. Se describió a la población entrevistada y se exploró la asociación de los componentes de la fatiga laboral con el bajo peso al nacer (BPN) mediante regresión logística, en un subgrupo de mujeres que laboraron en la venta ambulante durante su última gestación. Resultados. De las mujeres entrevistadas, $56 \%$ realiza jornadas de trabajo superiores a las 48 horas semanales, $87 \%$ carece de seguridad social y $68 \%$ tiene estudios no mayores de primaria. El riesgo de presentar BPN se eleva cuando se carece de control sobre la cantidad de mercancía a vender (RM 6.5, IC 95\% 1.3-31), se venden artículos de ocasión co mo refacciones y ornamentos (RM 6.3, IC 95\% 1.5-26), se exhibe la mercancía sobre el piso o se carga para su venta (RM 7.7, IC 95\% 1.8-32), y cuando los recursos para instalar el puesto por primera vez no proceden de la red social de apoyo de la vendedora (RM 7.4, IC 95\% 1.2-44). Conclusiones. Estos resultados contribuyen a identificar a las vendedoras con mayor riesgo de presentar un hijo con BPN, y sugerir medidas preventivas.

Palabras clave: condiciones de trabajo; fatiga; peso al nacer; vendedoras ambulantes; México

\author{
Hernández-Peña P, Kageyama ML, Coria I, \\ Hernández B, Harlow S. \\ Working conditions, labor fatigue \\ and low birth weight among \\ female street vendors. \\ Salud Publica Mex 1999;41:101-109.
}

\begin{abstract}
A bstract
Objective. This study presents the demographic, so cioeconomic profile, working conditions and labor fatigue among fertile age street vendors in Mexico City. Material and methods. 426 female street vendors were interviewed in Mexico City. This population was described and the association between the components labor fatigue and low birth weight (LBW) was analyzed by logistic regression in a subgroup of women who worked as street vendors during their last pregnancy. Results 0 f the interviewed group, $56 \%$ works mo re than $48 \mathrm{~h}$ per week, $87 \%$ had no social security, and $68 \%$ only had primary school level. The risk of LBW increased when workers had to cover selling quotes $(O R$ $6.5, \mathrm{C} 195 \% 1.3-31)$ when the merchandise were seasonal tools such as accesories or spare parts (O R 6.3, C 195\% 1.526), when women had to exhibit their merchandise on the floor or carry it (O R 7.7 C 195\% 1.8-32) and when financial support to initiate vending activities came from someone other than a close relative or friend (OR 7.4 C 195\%, 1.244). Conclusions. These results contribute to identify the female vendors with higher risk of having child with LBW and suggest preventive actions.
\end{abstract}

Key words: working conditions; fatigue; birth weight; street vendors; Mexico

* Este proyecto recibió financiamiento del Consejo N acional de Ciencia y Tecnología de México y de la Fundación Ford.
(1) Investigadora, Centro de Investigación en Salud Poblacional (C ISP), Instituto N acional de Salud Pública (IN SP), México.
(2) Jefa del Departamento de Bioestadística, CISP, IN SP, México.
(3) Jefa del Departamento de Estadística, Instituto N acional de Perinatología, México.
(4) Director de N utrición y Salud, CISP, IN SP, México.
(5) Investigadora, D epartamento de Epidemiología, School of Public Health, University of Michigan, Estados Unidos de América.

Fecha de recibido: 27 de febrero de 1998 • Fecha de aprobado: 18 de noviembre de 1998 Solicitud de sobretiros: Patricia Hernández-Peña. División del N orte 2741-2B, C oyoacán, 04030 México, D.F., México. 
$\mathrm{S}$ e ha documentado la asociación de las actividades laborales con los daños a la salud reproductiva. Los efectos negativos en la reproducción varían según el tipo de trabajo, la condición reproductiva y la situación particular de cada gestación. Entre las repercusiones más frecuentes se señalan el bajo peso al nacer (BPN), los abortos y las malformaciones congénitas. ${ }^{1}$

El estudio sistemático de las condiciones laborales asociadas potencialmente con el BPN se ha realizado a partir de la fatiga laboral, que incorpora, como elementos nocivos de una ocupación, la larga jornada laboral, las posturas mantenidas por tiempo prolongado, la operación de máquinas industriales, el esfuerzo físico, el estrés y algunos elementos de la exposición ambiental. ${ }^{2}$ La presencia de fatiga laboral se asocia con el BPN ya sea por la mayor frecuencia de partos prematuros $^{3}(\mathrm{PP})$, o bien, por el retardo en el crecimiento intrauterino entre los hijos de las mujeres trabajadoras. ${ }^{4}$

La fatiga laboral puede afectar al feto en razón del gasto energético y la reducción del flujo sanguíneo uterino, lo que conduce a una reducción del aporte de oxígeno y nutrientes al producto, en particular en presencia de desnutrición materna. ${ }^{5}$ La fatiga tiene un mayor impacto durante el segundo y el tercer trimestres del embarazo, al comprometer en mayor medida el crecimiento fetal. La modificación de los hábitos maternos durante el embarazo puede proteger al producto, en función del momento gestacional y el nivel de fatiga eliminado. De hecho, se ha encontrado una reducción en el riesgo de BPN en las trabajadoras que cuentan con licencia por maternidad. ${ }^{6}$

La fatiga laboral fue descrita inicialmente en el caso de las obreras del sector industrial, pero en Francia y Canadá también se ha analizado a partir de las ocupaciones sedentarias que se asocian con nacimientos prematuros. ${ }^{7}$ En México, la importancia de la fatiga laboral quedó demostrada entre un grupo de mujeres que trabajaba en la industria maquiladora, en cuyos hijos el BPN era más frecuente en comparación con aquellos cuyas madres trabajaban en los servicios y en el comercio. ${ }^{8}$ Los estudios de fatiga laboral y sus efectos en la salud reproductiva de trabajadoras de diversos servicios, como las taquilleras del Sistema de Transporte Colectivo Metro de la Ciudad de México ${ }^{9}$ las trabajadoras de servicios ${ }^{10}$ muestran que la fatiga laboral constituye un riesgo de BPN en las diversas ocupaciones de la mujer. En cada tipo de actividad, los componentes de la fatiga laboral asociados con el BPN son diferentes.
El BPN, además de estar relacionado con la fatiga laboral, se asocia con la edad materna extrema; la primiparidad y la multiparidad; los antecedentes reproductivos negativos, en particular el BPN en hijos previos, ${ }^{11}$ el estado nutricional materno y el aumento de peso durante el embarazo; el bajo nivel educacional y socioeconómico materno; la falta de atención prenatal, ${ }^{8}$ así como hábitos de vida nocivos, en particular el tabaquismo durante la gestación. ${ }^{6}$

La población que labora en actividades informales como la venta ambulante, no se beneficia de la protección que ofrecen las leyes laborales mexicanas. Las encuestas de ocupación informal muestran que sus jornadas de trabajo son prolongadas y que no cuentan con días de reposo; asimismo, esos trabajadores carecen de seguridad social, vigilancia de las condiciones de higiene y seguridad, vacaciones, retiro por maternidad y salario mínimo. ${ }^{12}$ La venta ambulante es una ocupación informal que se ha expandido en los últimos años y ha captado a la población femenina, principalmente durante su periodo fértil, por lo que representa una actividad que puede concentrar riesgo reproductivo. $^{12-14}$

El interés de este estudio es describir las características sociodemográficas, reproductivas y ocupacionales de las trabajadoras de la venta ambulante. Asimismo, se explora la asociación de la condición laboral con el BPN de los hijos de las vendedoras que laboraron en esta ocupación durante su última gestación.

\section{Material y métodos}

La investigación tuvo un diseño transversal. La información se captó en la Ciudad de México durante 1990, mediante una encuesta con vendedoras ambulantes que laboraban en las aceras. Las vendedoras ambulantes fueron definidas como mujeres que comerciaban cualquier tipo de bien en la vía pública.

Los criterios de inclusión del estudio fueron: ser vendedora ambulante y encontrarse en el puesto al momento de la visita, tener entre 12 y 50 años, haber tenido al menos un nacimiento durante los tres años previos al momento de la recolección de datos y aceptar participar en el estudio. Se entrevistaron vendedoras que trabajaban en los conglomerados de venta ambulante e independientes, ubicadas en las inmediaciones de dichos conjuntos de puestos. Se excluyeron los grupos institucionalizados de voceadores, vendedores de lotería y mercados sobre ruedas, ya que cuentan con prestaciones particulares, así como aquellas 
vendedoras que laboraban sólo uno o dos días por semana, por ser menor su exposición a los factores ocupacionales de riesgo.

Se obtuvo una muestra probabilística de vendedoras ambulantes de la ciudad. En 1990 existían 13500 concentraciones y 112081 puestos distribuidos en las delegaciones políticas de la ciudad. Se utilizó el marco muestral de vendedores ambulantes de ambos sexos elaborado por la Cámara Nacional de Comercio de la Ciudad de México. ${ }^{15}$ El muestreo fue aleatorio por conglomerados y multietápico. En la primera etapa se eligieron aleatoriamente por selección proporcional cuatro de las 16 delegaciones políticas de la Ciudad de México (Cuauhtémoc, Carranza, Iztapalapa e Hidalgo) y, dentro de ellas, las concentraciones de vendedores ambulantes.

En las concentraciones elegidas se recorrieron 5000 puestos entrevistando a las vendedoras ambulantes en edad fértil (1 874 mujeres), a fin de identificar a aquellas que cumplieran con los criterios de inclusión. Este último grupo quedó integrado por 426 mujeres (22.7\% del total), a quienes se aplicó el cuestionario completo.

La información se recolectó mediante un interrogatorio directo a las vendedoras. El cuestionario incluyó preguntas sobre las características de salud reproductiva (paridad, antecedentes reproductivos negativos y peso al nacer del último y penúltimo hijos), las condiciones sociodemográficas (edad de la entrevistada, características de su hogar y situación migratoria) y las condiciones económicas y laborales (escolaridad, mercancía, tipo de puesto, ubicación, ingreso mensual por la actividad y responsabilidad de la mujer en el aporte al gasto familiar). Para el estudio de la fatiga laboral se tomaron los componentes propuestos por Mamelle, ${ }^{16}$ adecuándolos a la venta ambulante. La presencia de cada uno de ellos entre las vendedoras se estableció de la siguiente manera:

1. Esfuerzo fisico. Se definió como el levantamiento de bultos de mercancía, la frecuencia del esfuerzo, el peso de la carga estimado por la mujer y la percepción de cansancio derivado de este esfuerzo.

2. Ambiente físico. Se evaluó a partir de la molestia generada por el ruido y el esmog, según la ubicación del puesto, y la percepción de la ocupación como nociva para la salud.

3. Jornada de trabajo. Se definió como el número de horas semanales dedicadas al trabajo, el tiempo diario de traslado desde el hogar al lugar de trabajo y de regreso, y las horas semanales de trabajo doméstico. Se indagó también la percepción de cansancio que la jornada laboral generaba.
4. Postura. Se investigó acerca de la permanencia diaria en cada postura durante la jornada laboral -caminando, parada, sentada o hincada- y la percepción del cansancio generado por la postura habitual.

5. Exposición a estresores laborales. Se indagó: la presencia de conflicto entre las vendedoras, ya fuera con su grupo, con otros grupos o con las autoridades; la percepción sobre la importancia de estos conflictos, y la presión laboral expresada como forma de pago, salarial o a destajo.

6. Control sobre la actividad laboral. Se determinó investigando la participación de la vendedora en decisiones comerciales y laborales relacionadas con la mercancía a vender, el precio, la ubicación del puesto, la cantidad de venta a realizar y el horario a cubrir.

7. Apoyo social. Se valoró la disponibilidad de apoyo social a partir de la presencia de familiares en la actividad, así como la ayuda para iniciarse en la ocupación, el tipo de ayuda y su procedencia.

El BPN se captó por medio del informe materno de bajo peso al nacer o por notificación de peso del producto inferior a 2500 gramos. No se discriminaron sus dos componentes: parto prematuro (PP) y retardo del crecimiento intrauterino (RCI). ${ }^{17}$

Las vendedoras ambulantes entrevistadas (426) se describieron a partir de las variables estudiadas. En una segunda etapa, del total de entrevistadas se seleccionó un subgrupo de 90 mujeres que habían trabajado como vendedoras ambulantes durante su última gestación, a fin de medir, mediante razones de momios (RM) e intervalos de confianza (IC), la asociación de algunas características relevantes con el BPN. Para controlar las variables potencialmente confusoras, tales como edad de la madre, paridad, atención prenatal, tabaquismo, escolaridad y el antecedente de BPN, se utilizó regresión logística no condicional. ${ }^{18}$

\section{Resultados}

En el cuadro I se presentan las características socioeconómicas y reproductivas de las 426 mujeres entrevistadas. La mayoría tenía entre 19 y 35 años. La edad promedio de las vendedoras -todas ellas en edad fértil- fue de 26 años; casi la mitad tenía entre dos y cuatro hijos, con un promedio de tres. El 68.5\% no realizó estudios o estudió hasta la primaria, y el resto de las mujeres estudió algún año de secundaria o más. La mayoría $(76.3 \%)$ vivía en la ciudad.

Sus condiciones laborales se muestran en el cuadro II. Más de la mitad de estas trabajadoras (56.8\%) 


\begin{tabular}{|c|c|c|}
\hline \multirow{2}{*}{\multicolumn{3}{|c|}{ Cuadro I }} \\
\hline & & Características SOCIOdEMOgRÁfiCAS \\
\hline \multicolumn{3}{|c|}{$\begin{array}{l}\text { Y REPRODUCTIVAS DE VENDEDORAS AMBULANTES. } \\
\text { MÉXICO, D.F., } 1990\end{array}$} \\
\hline Variable & Frecuencia & $\begin{array}{c}\% \\
N=426\end{array}$ \\
\hline \multicolumn{3}{|l|}{ Edad de la vendedora } \\
\hline 15-18 años & 30 & 7 \\
\hline $19-35$ & 351 & 82.4 \\
\hline 36 y más & 45 & 10.6 \\
\hline \multicolumn{3}{|l|}{ Paridad } \\
\hline 1 nacimiento & 129 & 30.3 \\
\hline $2-4$ & 210 & 49.3 \\
\hline 5 y más & 87 & 20.4 \\
\hline Abortos previos & 88 & 20.7 \\
\hline Ó bitos & 21 & 5.2 \\
\hline Hijos muertos & 37 & 8.7 \\
\hline Antecedente de hijo con BPN & 25 & 5.9 \\
\hline N unca asistió a control prenatal & 117 & 27.5 \\
\hline \multicolumn{3}{|l|}{ Escolaridad } \\
\hline Secundaria y más & 134 & 31.5 \\
\hline Primaria o menos & 292 & 68.5 \\
\hline
\end{tabular}

tenían jornadas que superaban las 48 horas de trabajo normadas por la Ley Federal del Trabajo como máximo semanal. La duración media de la jornada laboral semanal entre las vendedoras fue de 51.8 horas $(\mathrm{DE}=$ 19.1), con un mínimo de 5 y un máximo de 105 horas por semana. La duración de la jornada de trabajo no era regulada por $57.5 \%$ de las propias trabajadoras (cuadro III).

El tiempo de traslado de la casa al lugar de trabajo y de regreso varió entre dos minutos y siete horas; únicamente 10 de ellas requerían de periodos de traslado superiores a cinco horas por día (2.2\%), y 16 vendedoras tenían el puesto en la vía pública contigua a su hogar (3.8\%). Más de la mitad de las vendedoras necesitaba al menos 1.5 horas diarias para su traslado (cuadro II).

La jornada doméstica mostró un valor máximo de 70 horas por semana. La mitad de las vendedoras trabajaba en su hogar hasta tres horas por día. Solamente $3.8 \%$ no realizaba trabajo doméstico. De todas las vendedoras, $56.1 \%$ contaba con ayuda en sus quehaceres domésticos.

Durante la jornada laboral, $63 \%$ de las vendedoras permanecían sentadas durante periodos de hasta

\section{Cuadro II \\ Condiciones laborales entre las Vendedoras ambulantes. MÉxico, D.F., 1990}

Condiciones de trabajo

Frecuencia $\%$

Esfuerzo físico: carga 11 y más kg

329

77.2

Frecuencia del esfuerzo físico

\begin{tabular}{lrr} 
Moderado, escaso & 369 & 86.6 \\
\hline Frecuente & 57 & 13.4
\end{tabular}

Tiempo de traslado al puesto

\begin{tabular}{lll} 
Hasta 90 minutos por día & 157 & 36.9 \\
\hline 91 y más & 269 & 63.1
\end{tabular}

Postura sedente (hincada 0 sentada)

\begin{tabular}{lll} 
Hasta 4 horas por día & 270 & 63.4 \\
\hline Más de 4 & 156 & 36.6
\end{tabular}

Tiempo caminando

\begin{tabular}{lrr} 
Hasta 4 horas por día & 376 & 88.3 \\
\hline Más de 4 & 50 & 11.7
\end{tabular}

Duración de la jornada laboral

\begin{tabular}{lll} 
Hasta 48 hrs por semana & 184 & 43.2 \\
\hline 49 y más & 242 & 56.8
\end{tabular}

Percepción de la actividad

\begin{tabular}{lrr} 
Le molesta el esmog & 158 & 37.1 \\
\hline Le molesta el ruido & 161 & 37.8 \\
\hline Con problemas o peleas laborales & 63 & 14.8
\end{tabular}

Percepción de la postura

\begin{tabular}{lll} 
Cómoda & 253 & 59.4 \\
\hline Incómoda, muy incómoda & 173 & 40.7
\end{tabular}

$\begin{array}{lll}\text { Percibe largo su horario de trabajo } & 352 & 82.6\end{array}$

Percepción del esfuerzo físico

\begin{tabular}{lrr} 
N o, escaso & 221 & 51.9 \\
\hline Regular & 111 & 26.1 \\
\hline Grande & 94 & 22.1
\end{tabular}

cuatro horas por día (cuadro II). Menos de la cuarta parte realizaba su actividad caminando, y ésta ocurría durante periodos cortos (una a tres horas).

Más de la mitad de las mujeres $(60.3 \%)$ informaron que cargaban bultos de mercancía con un peso heterogéneo que, según estimaron, iba desde menos de $1 \mathrm{~kg}$ hasta $70 \mathrm{~kg}$; $77 \%$ cargaba más de $10 \mathrm{~kg}$. El esfuer- 


\section{Cuadro III \\ Control laboral y apoyo social entre las Vendedoras AMBULANTES. MÉXICO, D.F., 1990}

Control laboral $\quad$ Frecuencia $\quad \begin{gathered}\% \\ n=426\end{gathered}$

\begin{tabular}{lrl} 
Le indican dónde ubicar su puesto & 236 & 55.4 \\
\hline Le indican la mercancía a vender & 145 & 34 \\
\hline Debe cubrir cuota de mercancía a vender & 68 & 16 \\
\hline Le indican el precio de venta de su mercancía & 117 & 27.5 \\
\hline Le indican el horario de trabajo & 245 & 57.5
\end{tabular}

Apoyo social

Familiares en la ocupación

255

60

Financiamiento para instalar el puesto

por primera vez

\begin{tabular}{lrr} 
Recursos propios & 187 & 43.9 \\
\hline Préstamo de su red social & 91 & 21.4 \\
\hline O tro crédito & 148 & 34.7
\end{tabular}

zo de cargar los bultos se realizaba frecuentemente -más de tres veces por día- en $13 \%$ de las entrevistadas (cuadro II).

Casi 15\% de las vendedoras informaron tener conflictos en el trabajo por problemas con la policía, con su propio grupo o con el de otros vendedores ambulantes. Las condiciones de trabajo que la mujer percibió con mayor frecuencia como molestas fueron la duración de la jornada laboral (82.6\%) y la postura incómoda $(40.7 \%)$.

Más de la tercera parte de las mujeres percibían el ambiente laboral de manera negativa. Así, 41.5\% consideraba que su ocupación podía ser dañina y a $37.8 \%$ le molestaba el ruido; asimismo, a 37.1\% le resultaba incómodo el esmog (cuadro II). Las mujeres calificaron como más molesta la ubicación del puesto en el interior del metro y en los cruceros que en las aceras.

Las características del puesto se asocian con las condiciones en que la mujer realiza su actividad. Más de una tercera parte de las mujeres $(39.4 \%)$ cargaban su mercancía o la exhibían sobre el piso por carecer de infraestructura tubular; $36.2 \%$ se ubicaba al interior de las estaciones del metro o en los cruceros, y menos de la cuarta parte de las vendedoras $(23.2 \%)$ ofrecían mercancías de ocasión y de baja inversión, como refacciones automotrices y artefactos domésticos de ornato, entre otros.

El apoyo social y el control sobre las condiciones de trabajo resultaron elevados, pues $59.9 \%$ de las vendedoras tenían familiares en la misma ocupación, $55.2 \%$ fueron reclutadas por su red social de apoyo y
$22 \%$ recibieron ayuda material de dicha red para establecer el puesto. El financiamiento para iniciarse en la actividad, en casi la mitad de los casos, fue propio, y más de la tercera parte lo obtuvo fuera de su red social. En cuanto al control sobre las condiciones de trabajo, $44.6 \%$ lo tenía sobre la ubicación; $72.5 \%$, sobre el precio; $66 \%$, sobre la mercancía; $42.5 \%$, sobre el horario, y $84 \%$, sobre el monto de venta. El control resultó menor en el caso del horario de trabajo y la ubicación del puesto (cuadro III).

De las vendedoras, $74.2 \%$ dijo tener una pareja, que generalmente se encontraba trabajando al momento de la encuesta (70.4\%). En 25.8\% de los casos, la vendedora aportaba la mayor parte del gasto en el hogar, mientras que en el 31.9\% la aportación se hacía a partes iguales con el esposo. En conjunto, en $57.7 \%$ de los hogares, la mujer aportaba al menos la mitad del ingreso del hogar.

La gran mayoría de las vendedoras eran propietarias de la mercancía $(71.1 \%)$. Entre las no propietarias $(28.9 \%)$, las asalariadas fueron las más numerosas $(73.6 \%)$. La proporción de mujeres no remuneradas fue reducida $(9.1 \%)$. El resto $(17.4 \%)$ recibía ingresos por comisión.

De las mujeres, $61 \%$ indicó la presencia de algún síntoma durante su última gestación. Se registró una sintomatología múltiple en $37.1 \%$ de las mujeres (hipertensión, cefalea, sangrados, contracciones anormales, dolor lumbar e infecciones vaginales o urinarias). El 27.5\% de las entrevistadas no tuvo nunca una consulta prenatal. En los casos en que sí se recibió atención, $62.6 \%$ de las entrevistadas la recibieron en el primer trimestre de la gestación, $28.4 \%$ en el segundo, y una mínima proporción $(9.0 \%)$ no asistió a consulta sino hasta el tercer trimestre. Entre quienes recibieron atención prenatal, el número de consultas fue de hasta cinco en $46.2 \%$ de las mujeres, de hasta 10 consultas en una proporción similar de mujeres $(46.5 \%)$, y de más de 11 en $7.3 \%$ de los casos.

De las entrevistadas, $12.7 \%$ contaba con seguridad social. El hábito tabáquico resultó poco frecuente en esta población, pues sólo 8.7\% de las mujeres (34) informó que fumaba.

Entre los antecedentes reproductivos importantes, se identificó a 22 mujeres (5.2\%) que habían tenido algún nacido muerto. Ochenta y ocho mujeres (20.7\%) informaron haber tenido abortos previos, $5.9 \%$ refirieron antecedentes de BPN, y 12\%, de PP previos. Se identificó un total de 55 casos (12.9\%) de BPN en su último evento obstétrico, proporción que podría ser mayor dado que 55 casos notificaron desconocer el peso del hijo al nacer. Esta falta de información se presentó con mayor frecuencia entre quienes tenían menor esco- 
laridad y carecieron de atención prenatal y seguridad social, aspectos posiblemente asociados con el BPN (cuadro IV). Entre las 426 entrevistadas la prevalencia de BPN fue de $13 \%$.

La mayoría las mujeres $(77 \%)$ realizaba actividades remuneradas antes de su último embarazo, pero tan sólo 21.1\% trabajó durante la gestación. Su actividad en la época en que se realizó la entrevista era menor o igual de intensa que durante el embarazo, y en la tercera parte de las vendedoras era más intensa durante la gestación (34.3\%).

\section{Vendedoras expuestas a la actividad durante la gestación}

Entre las 90 mujeres que trabajaron durante su gestación en la venta ambulante, $14 \%$ presentó BPN; esta proporción no fue significativamente diferente de la encontrada en la población no expuesta (13\%).

La asociación de cada variable con el BPN se estudió en el subgrupo de 90 vendedoras expuestas a la actividad durante su última gestación, controlando por las variables que la literatura señala como asociadas a la presencia de BPN. Ya que la edad de la madre y la paridad estaban muy correlacionadas, se ajustó el efecto únicamente de la paridad y se incluyeron el tabaquismo, el antecedente de BPN en el hijo previo, la atención prenatal y la escolaridad. Considerando estos ajustes, en los casos en que el tiempo de traslado del domicilio al puesto y de regreso era superior a los 90 minutos, se encontró un riesgo 23 veces mayor de tener un producto con BPN. Este riesgo también fue 6.5 veces mayor para las vendedoras que deben cubrir una cuota de venta y que carecen de control sobre la cantidad de mercancía a vender; asimismo, en quienes carecen de infraestructura en el puesto y cargan la mercancía o la exponen sobre el piso para su venta, el riesgo fue 7.7 veces superior; entre las vendedoras de mercancía de ocasión como refacciones y objetos diversos, el riesgo de presentar un hijo con BPN resultó seis veces mayor que en las vendedoras de otros productos como alimentos, ropa y electrodomésticos, y en las vendedoras que requirieron de crédito para iniciar el negocio, cuando estos recursos no fueron proporcionados por la red social de apoyo, el riesgo fue siete veces superior. El antecedente de haber sufrido la muerte de algún hijo previo eleva ocho veces el riesgo de tener algún hijo con BPN (cuadro IV). El modelo con mayor ajuste conservó como variables asociadas el mayor riesgo de presentar BPN en este grupo de trabajadoras, el tipo de puesto, el tipo de mercancía y el origen del financiamiento, pero amplió aún más los intervalos de confianza, por lo que no se presenta aquí.

Para el grupo de vendedoras que permaneció en la actividad laboral durante algún momento de su última gestación, el análisis muestra que el esfuerzo físico de cargar los bultos de la mercancía no resultó ser relevante. Tampoco se encontró una asociación significativa de la jornada laboral con el BPN, salvo en lo relativo al tiempo de traslado, cuyo riesgo es elevado; sin embargo, dado el reducido tamaño muestral, el intervalo de confianza, aunque significativo, es muy amplio (RM 23.0; IC95\% 1.1-468.0).

\section{Discusión}

La venta ambulante se ha incrementado en el país durante las últimas décadas. Esta ocupación refleja uno de los principales cambios en el empleo femenino tanto de México como de la mayor parte de los países de América Latina. ${ }^{15}$ En efecto, según los datos que se obtuvieron aqui, una alta proporción de la población que se ocupa en el ramo está integrada por mujeres -se encontró que laboraban en $40 \%$ del total de puestos visitados-. La actividad laboral femenina durante la edad fértil es frecuente; según los datos obtenidos en este estudio $22 \%$ de ellas tuvieron un parto en los tres años previos. Esta es, de hecho, una tendencia univer-

\section{Cuadro IV \\ Variables asociadas al CONOCIMIENTO del PESO del Niño al NaCer, en vendedoras ambulantes de la CiUdad de México, 1990}

\begin{tabular}{|c|c|c|c|c|c|}
\hline \multirow[t]{2}{*}{ Indicador } & \multicolumn{2}{|c|}{ Conoce el peso } & \multicolumn{2}{|c|}{ Desconoce el peso } & \multirow[t]{2}{*}{$p$} \\
\hline & No. & $\%$ & No. & $\%$ & \\
\hline Sin atención prenatal & 83 & 70.9 & 34 & 29.1 & $<0.01$ \\
\hline Con atención prenatal & 288 & 93.2 & 21 & 6.8 & \\
\hline Sin seguridad social & 317 & 85.7 & 53 & 14.3 & 0.02 \\
\hline Con seguridad social & 54 & 96.4 & 2 & 3.6 & \\
\hline Primaria completa o menos & 242 & 82.9 & 50 & 17.1 & $<0.01$ \\
\hline Secundaria 0 más & 129 & 96.3 & 5 & 3.7 & \\
\hline
\end{tabular}




\section{Cuadro V \\ VARIAbles asociadas CON bajo PESO AL NACER EN MUJERES QUe trabajaron en la venta ambulante durante su embarazo. México, D.F., 1990}

Variable

$$
\frac{\text { Con BPN }}{\text { No. } \%}
$$$$
\frac{\operatorname{Sin} \mathrm{BPN}}{\mathrm{N} 0 .}
$$$$
\begin{aligned}
& \text { RM } \\
& \text { crudo }
\end{aligned}
$$

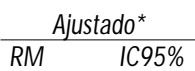

Hijos muertos previos

$\begin{array}{lccccccc}\text { No } & 9 & 11.3 & 71 & 88.8 & 1 & 1 & 1.5-42\end{array}$

Traslado al puesto

$\begin{array}{lccccccc}\text { Hasta } 90 \text { minutos } & 1 & 3.1 & 31 & 96.9 & 1 & 1 & 1.1-468\end{array}$

Control de la cantidad a vender

$\begin{array}{lccccccc}\text { Sí } & 8 & 10.5 & 68 & 89.5 & 1 & 1 & 1.3-31\end{array}$

$\begin{array}{lccccccc}\begin{array}{l}\text { Mercancía } \\ \text { Muebles, ropa }\end{array} & 6 & 9.5 & 57 & 90.5 & 1 & 1 & 1.5-26\end{array}$

Tipo de puesto

$\begin{array}{lccccccc}\text { Tubular, otro } & 3 & 5.3 & 54 & 94.7 & 1 & 1 & 1.8-33\end{array}$

Fuente de financiamiento

\begin{tabular}{llllllll} 
Propio & 2 & 5.6 & 34 & 94.4 & 1 & 1 & \\
\hline Red & 3 & 14.3 & 18 & 85.7 & 2.8 & 5.8 & $0.7-46$ \\
\hline 0 tro & 3 & 24.2 & 25 & 75.8 & 5.4 & 7.4 & $1.2-44$
\end{tabular}

$n=90$ mujeres

BPN : bajo peso al nacer

RM: razón de momios

*Ajustado por paridad, tabaquismo, atención prenatal, escolaridad, bajo peso al nacer antecedente

sal. En países como Estados Unidos de América (EUA), las mujeres embarazadas permanecen en su trabajo durante etapas cada vez más avanzadas de la gestación, condición que pudiera presentarse también en América Latina ${ }^{1}$ posteriormente. En este caso, sin embargo, la mayor parte de la población entrevistada se retiró de la actividad en algún momento del primer trimestre de embarazo.

Respecto a los cambios en la microeconomía doméstica asociados con el mayor empleo de la mujer, ${ }^{19}$ los datos corroboran que la mujer sigue conservando su función tradicional de maximizadora del ingreso al tiempo que se ha transformado en aportadora del gasto, pues al menos $50 \%$ de las vendedoras contribuyó con la mitad o más del ingreso del hogar, tanto en los hogares matrifocales como en los que existe pareja.
Las vendedoras ambulantes laboran en condiciones de informalidad, con largas horas de actividad, sin seguridad social y generalmente tienen una baja escolaridad, como se ha documentado en otros estudios. ${ }^{13}$

La frecuencia de BPN encontrada entre los hijos de las vendedoras resultó superior a la señalada para 1994 en la población no asegurada de la ciudad $(9.6 \%) .^{20}$ Más aún, la cifra encontrada podría estar subestimada por la probable presencia de sesgos de memoria en las entrevistadas, quienes en 55 casos no supieron informar sobre el peso de su hijo al nacer. En este último grupo coincidieron factores de riesgo que han sido asociados con el BPN, tales como la baja escolaridad y la ausencia de control prenatal. Se ha documentado que el informe materno sobre el peso al nacer de los hijos ha sido adecuado en madres con escolaridad prome- 
dio de secundaria, cuando se les entrevista en los tres años posteriores al nacimiento. Sin embargo, el mismo informe se ve afectado cuando el periodo se eleva a siete años. ${ }^{21}$ Los datos de este estudio sugieren que la menor escolaridad promedio de las vendedoras puede representar, también, una barrera para el informe adecuado de esta cifra por parte de la madre.

La permanencia de las vendedoras en la actividad durante su gestación no se asoció significativamente con el BPN, lo que pudiera atribuirse al reducido tamaño de la muestra en este subgrupo. Sin embargo, el punto de corte de la permanencia en la actividad laboral en la semana 28 de gestación permitió mostrar la existencia de un riesgo asociado con la presencia de BPN en el hijo. ${ }^{22}$

El número de mujeres entrevistadas que permaneció en la actividad durante la gestación es reducido. En ellas se constató una colinearidad entre las variables potencialmente asociadas a la presencia de hijos con BPN, por lo que se decidió excluir la edad materna. Se estudió el efecto de otras variables potencialmente confusoras como el antecedente de muertes previas y aborto. Sin embargo, no fueron incluidas en el modelo multivariado dado que el reducido tamaño de la muestra provocaba intervalos de confianza aún más amplios en las razones de momios ajustadas.

Los análisis ajustados por esas variables no modificaron la asociación entre las características de la venta ambulante y el BPN.

El estudio tiene algunas limitaciones que pueden afectar la interpretación de los resultados. El tamaño muestral obtenido es relativamente pequeño para el análisis de BPN. No obstante, cabe recordar que existen muy pocos estudios sobre salud reproductiva en trabajadoras informales. Por ello mismo, la descripción que se realiza en este documento representa un aporte exploratorio del problema.

La información sobre la variable dependiente y las de exposición procede del informe materno. La obtención de estimaciones cuantitativas entre las entrevistadas, como las horas de permanencia en una postura y el peso de los bultos que cargan, presentó dificultades. Empero, se considera que este error no difiere entre los casos con y sin BPN, por lo que únicamente disminuiría la fuerza entre las asociaciones encontradas. Otra limitación de este trabajo es la falta de información para calcular las probabilidades de selección de cada mujer, por lo que los estimadores de los errores de muestreo no fueron calculados de acuerdo con el diseño complejo de múltiples etapas.

Conviene recordar que el estudio y el embarazo ocurrieron en momentos diferentes. La discrepancia puede ser hasta de tres años. Una limitante más de este estudio es que la variable resultado de BPN pudo ser captada sólo a partir del informe proporcionado por la trabajadora, luego de un lapso que puede extenderse hasta tres años. Lo anterior puede generar problemas de mala clasificación y memoria. Tomando en cuenta estas limitaciones, la variable dependiente sólo se clasificó con dos categorías: presencia o ausencia de BPN.

El estudio de las condiciones ocupacionales de las vendedoras ambulantes adaptó el esquema de Mame$l^{2} \mathrm{e}^{2}$ a las características de esta actividad, y amplió los aspectos relativos al estrés, al control laboral y a la presencia de apoyos sociales para el trabajo, ${ }^{23}$ todas ellas características asociables a la actividad informal de la venta ambulante.

La mayor parte de las vendedoras desarrollan su jornada de trabajo en postura sedente. Aun cuando la postura sentada e hincada son fisiológicamente similares, cabe señalar que esta última se asocia probablemente con elementos culturales particulares que, por su baja frecuencia, no fueron analizados con mayor profundidad. Aun cuando otros estudios refieren el riesgo de BPN como asociado a una postura de pie por periodos superiores a siete horas,,$^{10}$ la postura en esta población no resultó relevante.

Una de las condiciones inherentes a la ocupación es la permanencia en la vía pública; sorprende que esta condición sea referida por menos de la mitad de las vendedoras como molesta por el ruido y el esmog.

La venta ambulante incluye el manejo de responsabilidades de índole «comercial», expresado en la toma de decisiones como un elemento de control del estrés y la fatiga. La ausencia de control sobre la cantidad a vender eleva el riesgo de que se presente BPN en esta población. La importancia del control sobre la actividad ha sido ya documentada en la literatura como asociada a una menor frecuencia de BPN en los hijos de trabajadoras negras gestantes de EUA ${ }_{;}^{24}$ asimismo, recientemente se ha identificado en mujeres de Carolina del Norte un riesgo de 1.4 (IC95\% 1.0-2.2) asociado con parto pretérmino, cuando el trabajo es de alta demanda y escaso control. ${ }^{25}$ La mayor parte de las vendedoras ambulantes dispone de redes sociales de apoyo en el ámbito laboral, mismas que facilitan su acceso y permanencia en el negocio, incluso cuando las mujeres carecen de experiencia laboral previa.

Mamelle ${ }^{2}$ identificó la asociación de la fatiga con el BPN en 1168 mujeres gestantes de 50 fábricas de Francia. Las razones de momios relevantes en su estudio de casos y controles fueron el trabajo con máquinas (RM 1.7, IC95\% 1.01-2.09) y el estrés (RM 1.54, IC95\% 1.03-2.3). En nuestra población, las variables relevantes fueron: la jornada de traslado, las caracte- 
rísticas del puesto, el tipo de mercancía en venta y el control sobre las condiciones de trabajo.

Otro estudio reciente ${ }^{26}$ identifica como factores de riesgo para el PP el estrés y la baja escolaridad, y para el RCI, el tabaquismo, el alcoholismo, la baja escolaridad y la ausencia de red social. Entre la población de este estudio, el tabaquismo era mínimo y el alcoholismo no se investigó.

El estrés, expresado como la presencia de conflictos en la actividad, no muestra asociación con la presencia de hijos con BPN. Se identificó un mayor riesgo cuando las vendedoras carecían de control sobre la cantidad de mercancía a vender y cuando habían recibido ayuda externa a su red social para iniciarse en el puesto. Estudios anteriores han mostrado una asociación del BPN con la pobreza, considerada a partir del ingreso familiar per cápita. ${ }^{27}$ En la población de este estudio, la carencia de recursos para iniciarse en la actividad resultó ser un indicador que también refleja el nivel socioeconómico y que se asocia al BPN.

Este trabajo sugiere la presencia de un subgrupo de población trabajadora con mayor riesgo de tener un hijo con BPN por los siguientes factores asociados a la venta ambulante: traslados diarios de más de una hora y media, ausencia de control sobre la cantidad a vender, venta de mercancía de ocasión, ausencia de infraestructura para exhibir la mercancía y carga de la mercancía o colocación de la misma sobre el suelo, así como necesidad de recurrir a ayuda financiera para iniciarse en el negocio.

\section{Referencias}

1. Luke B, Mamelle N, Keiyh L, Muñoz F, Minogue J, Papiernik E et al.The association between occupational factors and preterm birth: A United States nurse's study. Am J O bstet Gynecol 1995;173(3):849-862.

2. Mamelle N, Laumon B, Lazar P. Prematurity and occupational activity during pregnancy.Am J Epidemiol 1984;119(3):309-322.

3. Berhman RE. Preventing low birth weight: A pediatric perspective. J Pediatr 1985;107(6):842-854.

4. Robkim CS,Anderson HR, Bland JM, Brooke OG, Chamberlain G, Peacock JL. Maternal activity and birth weight:A prospective population-based study. Am J Epidemiol 1990;131:522-531.

5. N úñez-U rquiza RM. La placenta de madres desnutridas. Salud Publica Mex 1988;30(1):54-67.

6. Cerón $M, H$ arlow $S$, Sánchez $C$. The risk of prematurity and small for gestational age birth in Mexico City: The effects of working conditions and antenatal leave. Am J Public Health 1996;86(6):781-783.
7. Mamelle N , Bertucat I, Muñoz F. Pregnant women at work: Rest periods to prevent preterm birth? Pediatr Perinat Epidemiol 1989;3:19-28.

8. Denman C. La salud de las obreras de la maquila: el caso de Nogales, Sonora. En: De la Peña G, Durán JM, Escobar A, García de Alba J, comp. Crisis, conflicto y sobrevivencia, Guadalajara: Universidad de Guadalajara, 1990:229-255.

9. Garduño MA, Rodríguez J. La salud y la doble jornada: taquilleras del metro. Salud Problema 1990;20:41-45.

10. Cerón-Mireles S, Sánchez-C arrillo IC, H arlow S, N úñez-U rquiza. Condiciones de trabajo materno y bajo peso al nacer en la Ciudad de México. Salud Publica Mex 1997;39:2-10.

11.A lberman E. Low birthweight. En: Bracken M, ed. Perinatal epidemiology. N ueva York: 0 xford University Press, 1984: 86-98.

12. Instituto $\mathrm{N}$ acional de Estadística, Geografía e Informática-Secretaría del Trabajo y Previsión Social. Encuesta N acional de Micronegocios 1992. México, D.F.: IN EGI, 1994.

13. Secretaría de Programación y Presupuesto-Secretaría del Trabajo y Previsión Social. La ocupación informal en áreas urbanas 1976. México, D.F.: STyPS, 1979.

14. Instituto Nacional de Estadística, Geografía e Informática-Secretaría del Trabajo y Previsión Social. Encuesta N acional de Micronegocios 1994. México, D.F.: IN EGI, 1996.

15. Camara $\mathrm{N}$ acional de Comercio. La venta ambulante en la Ciudad de México. México, D.F.: C AN AC 0, 1987, 1988, 1989.

16. Mamelle N, Muñoz F. 0 ccupational working conditions and preterm birth:A reliable scoring system. Am J Epidemiol 1987;126(1):150-152.

17. Kramer M. D eterminants of low birth weight: Methodological assessment and meta-analysis. Bull W orld Health 0 rgan 1987; 65(5):663-737.

18. Lang JM, Lieberman E, C ohen A.A comparison of risk factors for preterm labor and term small-for-gestational-age birth. Epidemiology 1996; 7:369-376.

19. Garduño MA, Márquez M. La salud laboral femenina. A puntes para su investigación. En: Laurell AC, coord. Para la investigación en salud de los trabajadores. W ashington, D.C.: O PS, 1994:235-263.

20. Secretaría de Salud. Boletín de información estadística. México, D.F.: SSA, 1994.

21. G askin P,W alker SP, Forrester TE, G rantham-McG regor SM.The validity of recalled birthweight in developing countries. Am J Public Health 1997;87(1):114.

22. N aeye $R$, Peters E. Working during pregnancy: Effects on the fetus. Pediatrics 1982; 69(6):724-727.

23. Karasek $R$, Theorell T. Healthy work. Stress, productivity and the reconstruction of working life. N ueva York: Basic Books, Inc. Publishers 1990:1-47.

24. Hickey C, Cliver S, Mulvihill F, Mc N eal S, Hoffman H, Goldenberg R. Employment-related stress and preterm delivery: A contextual examination. Public Health Rep 1995;110(4):410-418.

25. Strogatz DS, Savitz DA. Employment, job strain and preterm delivery among women in N orth Carolina. Am J Public Health 1997;87:199-204.

26. N ordentoft M, Low G, H ansen D, N iver J, Pryds O, Rubin P et al. Intrauterine growth retardation and premature delivery. The influence of maternal smoking and psychosocial factors. Am J Public Health 1996;86(3): 347-354.

27. Rodríguez-Escudero FJ. The economic implications in pregnancy outcome. Int J Gynecol-O bstet 1981;19:231-237. 